\title{
Los procesadores de texto y los niños escritores: Un estudio de caso
}

\author{
Word Processors and Writing Children: A Case Study
}

\author{
Processadores de texto e filhos escritores: Um estudo de caso
}

\author{
Andrés Chiappe, ${ }^{a}$ Ana-Ruby González. ${ }^{b}$ \\ ${ }^{a}$ Universidad de La Sabana, Campus Universitario Puente del Común, Km 7 Autopista norte de Bogotá, \\ Chía, Cundinamarca, Colombia. Fono: 571-8615555 ext. 42303. \\ Correo electrónico: andres.chiappe@unisabana.edu.co \\ b Institución Educativa Técnica Agropecuaria, Viracachá. Mz 4 - C 16 Barrio Bolívar, Tunja, Boyacá, \\ Colombia. Fono: 571-7434135. Correo electrónico: gonzalezlopezruby@ gmail.com
}

\begin{abstract}
En este artículo se presentan los resultados de una investigación que tuvo por propósito identificar las transformaciones en la manera en que un grupo de estudiantes de grado segundo de básica primaria produjeron textos escritos, en el marco del uso del procesador de textos como instrumento complementario de escritura. Para abordar la producción de textos de estos estudiantes se tuvieron en cuenta cuatro categorías de análisis, a saber: Silueta del escrito, Signos de Puntuación, Conectores y Lingüística Oracional aplicada a la comunicación escrita. Los resultados del estudio permitieron identificar la pertinencia del uso del procesador de textos como instrumento complementario al cuaderno de escritura, para el desarrollo de habilidades para la producción de textos escritos a edades tempranas.
\end{abstract}

Palabras clave: escritura, informática educativa, computación y educación, desarrollo de habilidades, experiencias de aprendizaje.

\begin{abstract}
This article presents the results of a qualitative research carried out in order to identify changes in the way a group of second grade students of elementary school produced texts in the context of using the word processor as a complementary tool for writing. To address the production of texts of these students four categories of analysis were considered: Silhouette of writing, Punctuation Signs, Connectors, and Sentence Linguistics applied to written communication. The results of the study helped to identify the relevance of the use of the word processor as a complementary tool to hand writing, to develop skills for the production of written texts at an early ages.
\end{abstract}

Key words: writing, computer education, computing and education, skills development, learning experiences.

\section{RESUMO}

Apresentam-se os resultados de pesquisa realizada para identificar mudanças em textos produzidos no contexto de utilização do processador de texto como ferramenta complementar de escrita de um grupo de estudantes da segunda série do Ensino Fundamental. Para lidar com a produção de textos desses alunos consideraram-se quatro categorias de análise nomeadamente silhueta escrita, pontuação, conectores e Linguística oracional aplicadas à comunicação escrita. Resultados ajudaram na identificação da relevância da utilização do processador de texto como ferramenta complementar para escrita do livro, para desenvolver habilidades para a produção de textos escritos em uma idade precoce.

Palavras chave: escrita, educação em computação, computação e educação, desenvolvimento de competências, experiências de aprendizagem. 


\section{INTRODUCCIÓN}

Un niño que se destaque por la elocuencia en su escritura es reconocido por sus compañeros, su familia y la sociedad en la que vive, por lo cual resulta en extremo relevante fortalecer la lecto-escritura en los niños. Es importante que "el niño encuentre placer en interrogar y producir textos escritos, lo que contribuye al desarrollo auténtico de la comunicación y la realización personal" (Jolibert y Jacob, 1998: 9).

Por otra parte, una de las grandes preocupaciones actuales del sistema educativo tiene que ver con las dificultades manifiestas que presentan los estudiantes de distintos niveles para expresarse de manera escrita (Álvarez, 2012). En ese sentido, para la comunidad académica en general es de alta relevancia el fortalecimiento de sus habilidades tanto lectoras como de producción de textos escritos, sobre todo desde los primeros grados escolares.

En ese contexto, se trazó una investigación cuyo propósito se enfocó en explorar los aportes que pudieran brindar las TIC en el ejercicio de producción escrita en niños de segundo grado de educación básica primaria. La comprensión de dichos aportes y limitaciones constituye el primer paso para el diseño y planeación de estrategias didácticas apoyadas en TIC que persigan el fortalecimiento de las capacidades de lectura y escritura en nuestros niños.

\section{ABORDAJE TEÓRICO}

Es indudable que el ejercicio de escribir ha sido afectado por el uso de los computadores, hasta tal punto que la escritura electrónica ha empezado a concebirse no como una etapa tardía de la escritura impresa sino como un fenómeno relacionado pero independiente, con características y desempeños propios. Es así como "El texto se volvió más inmediato, más fragmentado y fluido y el medio ofrece mayor capacidad para la participación individual y la interactividad" (Ferris, 2002: 2).

Los efectos de la incorporación de las TIC por la vía del uso del procesador de textos ha sido en los años recientes un tema de interés para la comunidad investigativa y docente. Numerosas investigaciones se han trazado para indagar acerca de las relaciones entre el uso de las TIC y los procesos de lectura y escritura en niños y jóvenes en distintas partes del mundo. Sin el ánimo de agotar todas las posibles perspectivas que se han abordado sobre este tema, se mencionan algunos de los ejemplos más relevantes en los cuales se compara la escritura a mano con el uso de procesadores de texto en términos de las predilecciones de los estudiantes, diferencias en las habilidades de composición y procesos cognitivos (Burke \& Cizek, 2006; Dybdahl, Shaw \& Blahous, 1997; Ferris, 2002; Mogey \& Hartley, 2013; Mogey et al., 2012, 2010, 2008; Whithaus, Harrison \& Midyette, 2008). Otros investigadores y autores se enfocan en los cambios de las relaciones entre el escritor, el texto y el lector (Bolter \& Bolter, 1991; Grigar, 2005; Lyman, 1984; Moran, 1983; Takayoshi, 1996; Zinna, 2011), y otros puntualizan sobre el rol del procesador de palabras como herramienta instruccional o sólo como instrumento productivo (Bangert-Drowns, 1993; Collier y Werier, 1995; De Smet et al., 2012; Goldberg, Russell \& Cook, 2003; Lunsford, 2006; Morphy \& Graham, 2012; Pennington, 1991; Russell \& Haney, 1997; Scardamalia, Bereiter \& Steinbach, 1984; Zvacek, 1988). 
Los abordajes teóricos acerca de la escritura son muy diversos y complejos y la literatura en esta materia es extensa y muy rica. En ese sentido, se ha considerado la escritura desde al menos tres perspectivas bien distintas, una en la que lo importante es la escritura como producto, otra en la que se hace especial énfasis en el contexto como factor transversal de la escritura, y una última, en la que se ubica teóricamente esta investigación, que considera la escritura como proceso (Björk y Blomstrand, 2000; Caldera, 2003; Cassany, 1996). En ese orden de ideas, Flower \& Hayes (1996, 1981) y Sag-Legrán (2010) describen la escritura como un fenómeno cognitivo relacionado a lo que ellos llaman la "construcción del problema retórico" y a las operaciones de planificación, textualización y revisión.

$\mathrm{Al}$ respecto, Ferreiro y Teberosky indican que: "Hace ya tiempo que tanto psicólogos como educadores intuían que el aprendizaje de la lectura y la escritura no podía reducirse a un conjunto de técnicas perceptivo-motrices ni a la voluntad o a la motivación, pensando que debía tratarse, más profundamente, de una adquisición conceptual" (1999: 11).

De la misma manera, en una de sus obras anteriores mencionan que: "El acento en la reproducción de trazados reduce la escritura a un objeto "en sî́, de naturaleza exclusivamente gráfica; insistir en la correspondencia fonema-grafema, es presentar la escritura como "espejo" de los aspectos sonoros del lenguaje. Ni "refleja" sólo los fonemas, ni es un objeto "opaco". Es el producto de una construcción mental [...]" (Ferreiro y Teberosky, 1981: 10).

Desde el punto de vista de esta investigación se toma postura frente al tema y se reconoce que en el aprendizaje de la escritura, además de ser considerados aspectos mecánicos y procedimentales, se presenta un ejercicio de actividad y desarrollo cognitivo en el cual convergen múltiples factores que se vivencian y articulan natural y finalmente en el acto de escribir. "La lectura y la escritura son actividades de lenguaje y cognición profundamente relacionadas, que adquieren forma mediante el uso" (Langer Cit. en Guzmán, 2004: 161).

Para efectos de esta investigación, algunos de los aspectos del proceso de escritura que se conformaron como categorías de análisis se tomaron de los elementos formulados por Jolibert y Jacob (1998), los cuales se encuentran enmarcados en lo que hasta ahora se ha formulado como: silueta (correspondencia con el tema y aspecto general), signos de puntuación y ortografía, conectores y volumen de escritura.

La silueta del texto hace referencia a la superestructura del mismo, en otras palabras, quiere decir que el niño escribe sobre lo que debe escribir en cuanto a distribución espacial y los bloques de texto que lo conforman. Según Jolibert y Jacob, "si identificamos la silueta de una carta y por tanto la coherencia global podremos identificar un logro significativo en relación con el aprendizaje de la escritura" (1998: 71). Para determinar la coherencia global de cada producción escrita, en este parámetro se analizan dos aspectos: correspondencia con el tema y aspecto general del texto.

Por otra parte, además del uso adecuado de los signos de puntuación y la ortografía se consideran las omisiones, disociaciones, separaciones de palabras, inversiones y la mezcla de letras mayúsculas con letras minúsculas, pues se constituyen en los equívocos más comunes en el aprendizaje de la escritura en el nivel de los estudiantes involucrados en esta investigación.

En cuanto a los conectores, éstos se definen como elementos que ponen en conexión diferentes partes de un texto o diferentes textos. Dicho de otro modo, "El conector es una unidad que vincula un enunciado con otro elemento anterior, ya sea realmente proferido o simplemente accesible en el contexto" (Portolés, 1993:144). 


\section{MÉTODO}

La investigación se diseñó a manera de un estudio de caso de tipo exploratorio y naturaleza eminentemente cualitativa, y con el propósito de observar, documentar y analizar el proceso de producción de textos en niños de segundo grado de primaria. Dicha producción involucró el ejercicio de escritura a mano alzada y mediante el uso de procesadores de texto. Cabe anotar que la intención del estudio pese a su naturaleza comparativa no pretende establecer relaciones de causalidad directa entre el uso del procesador y el desarrollo de la competencia de escritura en los niños, sino identificar aquellos aspectos del proceso de escritura que evidencian cambios al momento de su uso en las aulas de clase.

Desde luego, y como se verá más adelante, algunos de estos cambios podrían deberse específicamente al uso del procesador de textos, pero en algunos otros, dada la naturaleza compleja de la escritura y del mismo desarrollo cognitivo de los niños y de su contexto (De Barrera, 2003), tal afirmación resultaría poco fundamentada e inclusive temeraria.

Con el fin de erradicar las deficiencias en comprensión y producción de textos que presentan los niños de segundo grado de básica primaria, los maestros en general se esfuerzan por aplicar diversas estrategias metodológicas (Olson et al., 2010; Regan \& Berkeley, 2012; Villalón et al., 2011), no todas ellas con resultados efectivos (Corcoran \& Silander, 2009). Para efectos de esta investigación, la estrategia didáctica involucró el uso de las Tecnologías de la Información y la Comunicación, más específicamente el uso del procesador de textos como instrumento complementario al cuaderno de escritura a mano alzada.

El uso directo del procesador de textos y del cuaderno de escritura se desarrolló de manera alternada de forma que se pudieran reducir al máximo las incidencias propias de la experiencia de escribir en un medio antes que en el otro. Por otra parte, las actividades de escritura en el cuaderno se adelantaron en el aula tradicional de clase y las que involucraron el uso del procesador de textos se adelantaron en la sala de computadores del colegio.

En cuanto a lo anterior, cabe anotar que las condiciones propias del espacio físico son similares en ambas aulas, por lo cual resulta improbable una injerencia particular del espacio en el cual se desarrollaron las actividades de producción de textos. No obstante ello, el uso de las salas de computadores para estos niños es muy esporádica, por lo cual en las primeras sesiones de trabajo el nivel de motivación por este aspecto fue alto dada la novedad, la cual fue tornándose normal a medida que fue pasando el tiempo. Lo anterior es consistente con lo que mencionan diversos autores con relación a que la motivación propia de las tecnologías es efímera (Area Moreira, 2010; Carrasco Pradas, Gracia Expósito y De la Iglesia Villasol, 2005; Cox, Cox \& Preston, 2000; Karsenti y Lira-Gonzales, 2011; Passey et al., 2004; Salinas Herrera, 2004; Tejada Fernández, Navío Gámez y Ruiz Bueno, 2007).

Cabe anotar que, de acuerdo a lo expresado hasta el momento, las actividades desarrolladas en la investigación se enmarcaron en los procesos u operaciones previamente mencionadas, a saber: la planificación textual, la textualización y finalmente la lectura y corrección del texto.

En la planificación textual, el estudiante se preparó bajo la orientación de la docente teniendo como recursos principales sus libros de Lengua Castellana y algunas presentaciones electrónicas. Luego de una gama posible de temas propuestos tanto por la docente como 
por los niños, cada uno de ellos seleccionó libremente el tipo de texto y el tema con el cual sintiera especial goce al escribir. Además, se formularon varios interrogantes acerca de lo que se pensaba escribir para saber qué conocimientos poseían y aplicar lo aprendido en la clase. La operación siguiente fue la textualización, la cual consistió en escribir utilizando el procesador de textos y su cuaderno de escritura. La operación de corrección consistió en dar lectura al escrito y modificar lo que se considerara que no correspondía al texto; para ello los niños utilizaron las diversas herramientas que proporcionan los equipos de cómputo, las sugerencias hechas por sus compañeros y por la docente encargada del curso.

\subsection{PARTICIPANTES}

La población con la que se trabajó la investigación fueron los niños de segundo grado de básica primaria. El grupo estaba conformado por 7 niñas y 12 niños, de edades comprendidas entre los 7 y 10 años, hijos de agricultores y comerciantes de la región. Un $16 \%$ de los participantes tenía computador en el hogar y podía acceder a Internet con ayuda de un adulto. Los demás niños utilizaron los computadores del colegio. Todos los niños participantes tenían un manejo básico del computador, es decir, se les facilitaba interactuar con programas sencillos, como juegos y el procesador de textos. Con la selección de los participantes se buscó la mayor continuidad posible en términos de tiempo de interacción, con lo cual se aseguró la permanencia del grupo por un período de un año, tiempo en el cual se realizaron las distintas actividades de producción de textos analizadas en esta investigación.

\subsection{DURACIÓN DE LA INVESTIGACIÓN}

El estudio se realizó durante dos años y se trazó a través de cinco diferentes fases o etapas. La primera de ellas se desarrolló durante seis meses bajo un enfoque de exploración teórica, de rastreo de antecedentes sobre el objeto mismo de la investigación y de diagnóstico inicial para identificar la manera en que los niños producían sus textos escritos. El diagnóstico arrojó la siguiente información: el 26\% de los niños presentó dificultades en su motricidad fina, su producción de texto era escasa y sus avances en escritura eran muy lentos. De todo el grado, un 57\% presentaba un número importante de disociaciones, separación de palabras, mezcla de mayúsculas y minúsculas, rotaciones e inversiones u omisiones.

En la segunda etapa se diseñaron e implementaron las actividades de producción de textos ya sea en su cuaderno de escritura como a través del uso de procesador de textos, actividades que se desarrollaron a lo largo de un año. Durante este período se realizó un pilotaje de estas actividades, y una implementación posterior sobre la base de los aprendizajes derivados de dicho pilotaje. La prueba piloto confirmó buena parte de la información encontrada en el diagnóstico inicial; mostró tanto las debilidades que presentaban los niños del grado primero en esta competencia comunicativa, como la etapa de apropiación de la escritura en la que se encontraban. Solucionar en gran medida estas deficiencias marcó el derrotero posterior de la investigación, ajustando las actividades de producción de textos que se realizarían en clase.

En la etapa de producción de textos cada niño participante generó 13 textos escritos en el cuaderno de escritura y 13 textos utilizando el procesador de textos de manera alternada, 
es decir, en algunas oportunidades se escribió antes en el cuaderno y luego en el procesador de textos y viceversa. Cada una de las parejas de estos 13 textos coincidió con un tema asignado dentro del cronograma establecido para el desarrollo de estas actividades en el marco del plan de estudios de Lengua Castellana para el grado segundo. El hecho de escribir en el cuaderno y en el procesador de textos sobre los mismos temas con algunas pequeñas variaciones y por la misma diversidad de los temas, minimizó la posibilidad de que los resultados comparativos en la escritura pudieran ser producto de la afinidad o conocimientos previos sobre algunos de los temas. Lo anterior conformó un cuerpo documental consistente en 494 textos, sobre los cuales se aplicaron las distintas categorías de análisis.

Los temas sobre los cuales se realizó la producción escrita fueron: la narración y la comunicación, la lírica y el uso de letras mayúsculas, el uso del punto y coma y la oración, los sinónimos y la historieta, los antónimos y el uso de las palabras con MP y MB, el diccionario y la sílaba, la descripción y los juegos de palabras, la televisión y el sustantivo, la carta y el adjetivo, el verbo y los aumentativos y diminutivos, la radio y los libros, el significado de las palabras y los conectores, y el párrafo y el uso de z, c.

La cantidad estimada de productos textuales generada por los niños participantes se considera suficiente para identificar algunas diferencias en su producción textual, teniendo en cuenta que no todos los niños se encuentran en la misma etapa de apropiación de la escritura, sus ritmos de aprendizaje no son homogéneos y las experiencias de cada uno de los niños con las TIC son diferentes.

Finalmente, se generó una última fase de análisis de la información encontrada, en la cual se llevaron a cabo acciones relacionadas con la codificación y recuperación de los textos producidos por los niños participantes y la generación de matrices de análisis y observación. En dichas matrices, dos observadores registraron, como parte de un proceso de análisis de contenido, la presencia de evidencias acerca de las categorías de análisis planteadas para esta investigación (silueta, signos de puntuación y ortografía, conectores y volumen de escritura). El coeficiente Kappa de Cohen aplicado a los registros de identificación de evidencias para los dos observadores fue de 0,61, lo cual permitió establecer un nivel satisfactorio de concordancia entre dichos procesos de observación.

\section{RESULTADOS Y DISCUSIÓN}

\subsection{RESUlTADOS EN CUANTO A LA SILUETA DEL TEXTO}

Tal como se adelantó, la silueta hace referencia a la superestructura del texto, en otras palabras, indica si el niño escribe sobre lo que debe escribir en cuanto a distribución espacial y los bloques de texto que la conforman. Según Jolibert y Jacob (1998), si se logra identificar la silueta de un texto y, por ende, su coherencia global, se podrá identificar un desarrollo importante en la competencia de producción de textos. Para determinar la coherencia global de cada producción escrita, en este parámetro se analizaron dos aspectos: correspondencia con el tema y aspecto general del texto.

El aspecto general del escrito forma parte de la silueta del texto e indica la apropiación de la escritura que el estudiante alcanzó en cada tema. Para caracterizarla de manera cualitativa se estableció la siguiente escala de valoración: 
Tabla 1. Criterios de valoración de la silueta del texto

\begin{tabular}{|c|c|c|c|c|}
\hline VALORACIÓN & ВАJO & BÁSICO & ALTO & SUPERIOR \\
\hline ABREVIATURA & B & BS & $\mathbf{A}$ & $\mathbf{S}$ \\
\hline $\begin{array}{l}\text { Criterio de desempeño } \\
B\end{array}$ & \multicolumn{4}{|c|}{$\begin{array}{l}\text { - No escribe el texto. } \\
\text { - Se entiende poco de lo que escribe y presenta demasiados errores } \\
\text { ortográficos y su producción es muy escasa. } \\
\text { - No hay correspondencia con el tema. }\end{array}$} \\
\hline $\begin{array}{l}\text { Criterio de desempeño } \\
B S\end{array}$ & \multicolumn{4}{|c|}{$\begin{array}{l}\text { - Hay correspondencia con el tema, pero su texto es escaso y poco } \\
\text { legible. } \\
\text { - Le falta argumentar y profundizar más sobre el tema. } \\
\text { - Presenta errores ortográficos. }\end{array}$} \\
\hline $\begin{array}{l}\text { Criterio de desempeño } \\
\text { A }\end{array}$ & \multicolumn{4}{|c|}{$\begin{array}{l}\text { - Hay correspondencia con el tema. } \\
\text { - Su texto es legible pero escaso. } \\
\text { - Sus errores ortográficos son mínimos. }\end{array}$} \\
\hline $\begin{array}{l}\text { Criterio de desempeño } \\
S\end{array}$ & \multicolumn{4}{|c|}{$\begin{array}{l}\text { - Hay correspondencia con el tema. } \\
\text { - Su producción escritural es completa y coherente. } \\
\text { - Escribe de manera convencional. } \\
\text { - Redacta, explica y demuestra interés frente al tema. }\end{array}$} \\
\hline
\end{tabular}

Fuente: elaboración propia.

A continuación se muestra a manera de ejemplo una de las matrices de análisis, para este caso la correspondiente a la silueta.

Tabla 2. Ejemplo de matriz de resultados (silueta del texto)

\begin{tabular}{|l|c|c|c|c|c|c|c|c|c|}
\hline TEXTOS & \multicolumn{4}{|c|}{ Observador 1 } & \multicolumn{5}{c|}{ Observador 2 } \\
\hline & B & BS & A & S & B & BS & A & S & Total \\
\hline Textos cuaderno & 48 & 63 & 96 & 40 & 53 & 68 & 91 & 35 & $\mathbf{2 4 7}$ \\
\hline Textos procesador & 36 & 88 & 89 & 34 & 41 & 93 & 84 & 29 & $\mathbf{2 4 7}$ \\
\hline
\end{tabular}

Fuente: elaboración propia.

Los resultados favorables en la escala alto y superior en la producción de textos utilizando el procesador, sumados a los resultados básico y bajo de la producción de textos 
en el cuaderno muestran, en una primera e interesante instancia, que las características de la silueta se logran de mejor manera cuando los estudiantes utilizan el procesador de textos, aunque cabe anotar que la magnitud de estas diferencias es comparativamente leve. Las observaciones realizadas permiten inferir que ante la posibilidad de corregir más fácilmente los errores en la escritura con el uso del procesador de textos, no sólo el volumen de escritura aumenta sino que se permite ajustar más fácilmente la correspondencia con el tema en el escrito, es decir, que el estudiante tiene más tiempo y oportunidad para repasar o reescribir. Haciendo una indagación más detallada sobre los casos de los niños en que la silueta del texto elaborado con el procesador indicó un resultado menor, se pudo establecer que dicho resultado se debió a varios factores, por ejemplo, que tuvieron un ritmo de aprendizaje lento o que no entendieron el tema y, desde luego, si no hay comprensión del tema, no se puede esperar que la producción escrita sobre dicho tema presente correspondencia con ella.

\subsection{RESULTADOS EN CUANTO AL PROCESO DE TEXTUALIZACIÓN: SIGNOS DE PUNTUACIÓN Y ORTOGRAFÍA}

La segunda operación que plantea Jolibert y Jacob (1998) en su modelo teórico sobre producción de texto es la textualización. En esta investigación se han tenido en cuenta dos de sus aspectos principales: la Lingüística Textual y la Lingüística Oracional y, dentro de ellas, se delimitó el análisis a los signos de puntuación, ortografía, los conectores y algunos de los errores más comunes de escritura en este nivel.

Para el análisis de estas categorías se elaboraron 66 matrices, en donde se registró la información en cada texto producido tanto con el procesador como en el cuaderno de escritura. Para facilitar su abordaje, el análisis se dividió en tres partes. En la primera parte se comparó si el estudiante utilizó en sus escritos mayúscula inicial, punto final y signos de puntuación, como la coma.

En la segunda parte, para facilitar el análisis se compararon seis dificultades comunes que presentan los estudiantes en sus escritos, como son: separa palabras, une palabras, omite letras, mezcla mayúsculas y minúsculas, hace inversión de letras, y omite y agrega $\mathrm{h}$.

En la tercera parte, se tuvieron en cuenta diez problemas principales de rotaciones que presentan los estudiantes en su escritura, y se hicieron anotaciones de otros errores ortográficos de cada estudiante en forma individual, los cuales son: i x y, s x z, v x b, b x v, c x s, s x c, b x d, y x ll, 11 x y, m x n.

Parecería inquietante pensar que funcionalidades del procesador de textos tales como la autocorrección pudieran haber incidido en el desarrollo de las actividades y en los resultados obtenidos en cuanto a la falta de punto o comas, rotaciones o utilización de mayúscula inicial, sin embargo, la autocorrección no influyó en el número de errores porque previamente a su uso se deshabilitó esta herramienta y sólo se utilizó bajo supervisión de la docente y en muy pocas ocasiones.

Los resultados muestran que los estudiantes, al utilizar el procesador de textos, hacen un mejor uso tanto de la mayúscula inicial, como del punto final y de la coma. En los textos escritos con el procesador hubo un incremento en el uso de la mayúscula inicial del $16 \%$, del punto final del $32 \%$ y de la coma un $76 \%$.

Cabe anotar que aquellos niños que evidenciaron un uso elevado de la mayúscula inicial, el uso de la coma y del punto final en el cuaderno de escritura, mantuvieron e inclusive incrementaron levemente esta práctica cuando hicieron uso del procesador de textos. 
La niña $N^{\circ} 7$ mejoró su producción de texto y su rendimiento al escribir. Se interesó para que las palabras le quedaran bien escritas; había aprendido que si una palabra estaba subrayada con rojo no era correcta y procedía a corregir el error de acuerdo a las sugerencias que le brindaba el procesador de textos, colocando el puntero del mouse y dando clic derecho; pero si no lograba dar solución al problema le preguntaba a la docente. [Segmento extraído del diario de campo].

Observaciones como la anterior permiten inferir que además de los asuntos de naturaleza técnica relacionados con el uso del procesador de textos o los relacionados con el desarrollo de la motricidad fina recreadas en la escritura en el cuaderno, los avances en su calidad se deben adicionalmente a factores motivacionales intrínsecos, es decir, que corresponden en su mayoría a estudiantes que se interesan por presentar un texto elaborado con calidad y con un mínimo de errores.

\subsection{RESULTADOS EN CUANTO A LA TEXTUALIZACIÓN: OTRAS PRINCIPALES DIFICULTADES DE ESCRITURA}

Para realizar este análisis se compararon los resultados de las dificultades más frecuentes en la textualización de la escritura que presentaron los estudiantes en los primeros años de vida escolar, con más frecuencia, agrupados de la siguiente forma.

\subsubsection{En cuanto a las omisiones}

Según Sánchez (2006), las omisiones se presentan cuando los niños suprimen letras en el momento de escribir. En ese sentido, los resultados muestran que el total de omisiones presentadas en los textos producidos con el procesador se redujo sólo en un $8 \%$ en comparación con las producciones generadas en el cuaderno de escritura. Lo mismo sucede con los problemas que presentan los estudiantes con la letra h, que en la gran mayoría se dan por causa de las omisiones y una mínima parte por agregarla en palabras que se escriben $\sin \mathrm{h}$. En términos generales, al utilizar el procesador de textos este problema sólo se reduce en un $5 \%$.

\subsubsection{En cuanto a las disociaciones}

Una disociación se presenta cuando se separan las silabas de una palabra y éstas se unen con la siguiente palabra (Prieto Cortés, 2012). Los hallazgos encontrados en este estudio indican que en la mayoría de los casos en que se presentan uniones de palabras, se presentan igualmente disociaciones. No obstante, este fenómeno se reduce en gran medida (30\%) cuando la escritura se realiza utilizando los procesadores de texto.

El conteo de palabras encontradas en las cuales se realizó separación de las sílabas que conforman las palabras, mostró una disminución del 69\% cuando los niños participantes escribieron utilizando el procesador de textos.

\subsubsection{En cuanto a la mezcla de letras mayúsculas con letras minúsculas}

Según observaciones reportadas en el diario de campo, a través de una verificación posterior a la producción de los textos, se logró identificar que la mayoría de las dificultades 
relacionadas con la mezcla de letras mayúsculas y minúsculas en el ejercicio de escritura en el cuaderno se presentaron en los niños que no habían establecido claramente la diferencia entre la letra mayúscula y la minúscula. Por otra parte, cuando la escritura se realizó mediante el uso de procesador de textos estas dificultades se presentaron más por falta de atención o concentración al momento de realizar el cambio con el botón de Bloqueo de Mayúscula en el teclado. Según los datos recogidos en la producción de textos de los niños participantes, este problema fue superado ampliamente en más de la mitad de los casos con el uso del procesador de textos.

\subsubsection{En cuanto a las rotaciones}

Una de las dificultades más comunes observadas en la escritura de los niños participantes son las rotaciones. Las rotaciones se presentan cuando "Los niños confunden algunas letras con otras que son semejantes" (Sánchez, 2006: 31). Las manifestaciones más frecuentes encontradas en las producciones de texto fueron las siguientes: $\mathrm{s} \mathrm{x} \mathrm{z,} \mathrm{b} \mathrm{x} \mathrm{v,} \mathrm{v} \mathrm{x} \mathrm{b,} \mathrm{c} \mathrm{x} \mathrm{s,} \mathrm{s} \mathrm{x}$ c, d x b, b x d, y en menos ocasiones se hallaron: ll x y, y x ll, i x y, n x m, y rr x r.

Los resultados muestran que los estudiantes disminuyeron estas dificultades con el uso del procesador de textos en la siguiente cuantía: y x i en $25 \%$, b x v en $28 \%$, v x b en $29 \%$, s x z en $25 \%$, b x d en 59\%, r x rr en $42 \%$, ll x y en 58\%, c x s en 57\%, y sólo aumentaron para el caso de la rotación s x c en $32 \%$ y n x m en $62 \%$. Se presume que el aumento en la rotación de estas dos últimas letras se debe a la cercanía de los dos caracteres en el teclado.

\subsection{RESULTADOS EN CUANTO A LA TEXTUALIZACIÓN: CONECTORES Y LINGÜÍSTICA ORACIONAL}

En este parámetro se analizó tanto la cantidad de conectores utilizados en los textos como el volumen de escritura, en términos de la cantidad de oraciones escritas. Los conectores son elementos que ponen en conexión diferentes partes de un texto o diferentes textos y establecen relación entre las oraciones. Aunque existen numerosos tipos de conectores, en este estudio sólo se han tenido en cuenta doce de ellos, los cuales corresponden al nivel de escritura propio de los niños de segundo grado de básica primaria.

El número total de los conectores empleados en los textos generados en el cuaderno de escritura fue $20 \%$ superior al de los textos elaborados con el procesador. Así mismo, se observó que en el primer escrito en el cuaderno los niños utilizaron 172 conectores en promedio, cifra muy superior a la de su último escrito que fue de 78. En los escritos elaborados con el procesador de textos los niños utilizaron tanto en el primer como en el último escrito casi el mismo número promedio de conectores: 102 y 101, respectivamente. Los conectores más utilizados en las dos producciones de texto fueron la conjuntiva " $y$ "; las disyuntivas "que", "porque"; las temporales "después", "entonces" y "cuando"; las aditivas "también", "además" y la comparativa "como".

En cuanto al volumen de escritura, el total de oraciones fue mayor cuando los estudiantes escribieron en sus cuadernos; en total emplearon 968 oraciones en comparación a las 823 oraciones escritas usando el procesador de textos.

El análisis general de los escritos y las observaciones de los productos de los estudiantes indican que la diferencia en el volumen de escritura se debe, en la mayoría de los casos, a que cuando se escribe con el procesador de textos hay menos redundancia en los escritos. 


\section{CONCLUSIONES}

Los resultados de este estudio llevan a considerar, a manera de reflexión general, la pertinencia de incluir decididamente la incorporación de las TIC, más específicamente del procesador de textos, en los primeros años de escolaridad, como componente de complementariedad al ejercicio de la escritura con lápiz y papel.

La complementariedad en mención incluye seguir haciendo énfasis en la importancia del desarrollo motriz del niño asociado al aprendizaje de la escritura, pero potencializada por las funcionalidades de un ejercicio adicional que haga uso de herramientas tan sencillas pero tan potentes como el procesador de textos.

No se trata entonces de promulgar un cambio extremo en las maneras de enseñar la escritura a los niños reemplazando los medios tradicionales por tecnologías digitales, sino combinarlas estratégicamente en función de generar nuevas posibilidades de enseñanza de la escritura.

Ahora bien, en términos generales, es muy difícil establecer una única causa de naturaleza técnica asociada al uso del procesador de palabras como mediación del proceso de escritura en los niños participantes. Los resultados indican, tal como lo menciona Cochran-Smith (1991), que son múltiples los factores que podrían haber generado buena parte de los resultados con relación a las transformaciones encontradas en la producción de textos, toda vez que la actividad escritora en los niños se encuentra transversalizada por factores de naturaleza didáctica, social, así como tecnológica y emocional. De manera que "El orden de progresión de conductas no impone, de hecho, un determinado ritmo en la evolución. Aquí, como en otros campos del desarrollo cognitivo encontramos grandes diferencias individuales" (Ferreiro y Teberosky, 1981: 2).

Asimismo, cabe realizar algunas inferencias en función de las distintas categorías de análisis trabajadas a lo largo de la investigación. En ese orden de ideas los cambios en cuanto a la silueta del texto son factibles debido a la capacidad de estandarización propia del procesador de textos, la cual se traduce en productos organizados en bloques de texto y aspecto agradable a la vista. No obstante, tal y como se identificó en los resultados, conseguir estos productos ordenados no sólo es un asunto relacionado con los aspectos técnicos de la escritura, sino por aspectos de tipo motivacional.

Lo anterior implica que el uso de herramientas tecnológicas por sí mismas no generan valor agregado con relación al mejoramiento del aspecto general de una producción textual, sino que más bien se presentan como agentes coadyuvantes que aprovechan una buena disposición hacia una escritura ordenada y consistente, y la ayudan a concretar.

Por otra parte, los correctores ortográficos pueden ser considerados como inconvenientes en el aprendizaje de la escritura si ésta se concibe como producto. Sin embargo, al ser considerada como proceso y relacionada con el ejercicio de operaciones cognitivas (Cassany, 1996; Flower y Hayes, 1996; Scardamalia, Bereiter \& Steinbach, 1984), los correctores se convierten en alarmas que le permiten al estudiante no sólo reconocer dichos errores, sino aprender de ellos, encontrar patrones en estos errores dentro de una página y reconocer sus propias dificultades en esta materia, a manera de ejercicio metacognitivo.

El manejo ortográfico del escrito mejora cuando los niños escriben haciendo uso del procesador de textos debido a las alarmas dispuestas por las herramientas informáticas, tanto desde la dimensión de software como desde el hardware. En ese sentido, la funcionalidad de alarma que proporciona el software de procesamiento de textos con 
relación al subrayado en color rojo de las palabras mal escritas es uno de los factores que les permite a los niños advertir los errores ortográficos y corregirlos de manera oportuna. Como el procesador no genera autocorrección automática, función que debe estar siempre desactivada, los niños que sistemáticamente escriben palabras con errores ortográficos son advertidos por el programa sobre sus errores de manera que la corrección de dichas palabras se realiza de manera reiterada, lo cual finalmente disminuye los problemas de omisiones, disociaciones, separación de palabras e inversión de letras. Con relación a la mezcla de mayúsculas y minúsculas, es la alarma visual del teclado que indica la fijación o bloqueo de las mayúsculas la que induce a la corrección de este tipo de errores. Algunas rotaciones se presentan por la cercanía de algunas de las letras en el teclado del computador, por ejemplo, m y n, s y z, y v y b.

Por otra parte, a diferencia de los textos elaborados en el cuaderno de producción, aquellos generados mediante el uso del procesador de textos tienen una utilización más adecuada de conectores. Lo anterior se presenta debido a que los niños no tienen que volver a digitar de nuevo todo el texto, sino que corregir sólo los segmentos que presenten errores en la producción textual.

En cuanto a la lingüística oracional del escrito, cuando los niños escriben utilizando el procesador de textos las transformaciones se expresan finalmente en el volumen de escritura, evidenciando una disminución en el número de oraciones escritas. Lo anterior es ocasionado por la facilidad correctiva de los procesadores de textos, funcionalidad que le permite a los niños tener más tiempo para reelaborar sus oraciones, omitir las frases que expresen lo mismo y complementar las oraciones que no tengan sentido completo, posibilitando entonces que haya menos redundancia. Así mismo, al utilizar el procesador de textos el estudiante puede visualizar en el monitor de su computadora su escrito elaborado con letra legible y en el tamaño que desee, lo que le facilita identificar segmentos mal redactados.

Por último, el uso del procesador de textos fortalece los procesos de revisión en la medida en que, a diferencia de la realizada sobre el papel, le permite al estudiante hacer fácilmente y de manera reiterada, si fuese necesario, modificaciones del texto dentro del proceso de revisión. Lo anterior es consistente con lo mencionado en esta materia por Caldera, cuando menciona que dentro de las estrategias de revisión se encuentran las que permiten "Dominar diversas formas de rehacer o retocar un texto: eliminar o añadir palabras o frases, utilizar sinónimos, reformulación global” (2003: 366).

\section{REFERENCIAS BIBLIOGRÁFICAS}

Álvarez, G. (2012). New Technologies in the University Context: The Use of Blogs for Developing Students' Reading and Writing Skills. Universities and Knowledge Society Journal, vol. 9, n. 2, 185-199.

Area Moreira, M. (2010). El proceso de integración y uso pedagógico de las TIC en los centros educativos: Un estudio de casos. Revista de Educación, n. 352, 77-97.

Bangert-Drowns, R. L. (1993). The Word Processor as an Instructional Tool: A Meta-Analysis of Word Processing in Writing Instruction. Review of Educational Research, vol. 63, n. 1, 69-93.

Björk, L. y Blomstrand, I. (2000). La escritura en la enseñanza secundaria: Los procesos del pensar y del escribir. Barcelona: Graó.

Bolter, J. D. \& Bolter, J. D. (1991). Writing Space: The Computer, Hypertext, and the History of Writing. Hillsdale: Erlbaum Associates. 
Burke, J. N. \& Cizek, G. J. (2006). Effects of Composition Mode and Self-perceived Computer Skills on Essay Scores of Sixth Graders. Assessing Writing, vol. 11, n. 3, 148-166.

Caldera, R. (2003). El enfoque cognitivo de la escritura y sus consecuencias metodológicas en la escuela. Educere, vol. 6, n. 20, 363-368.

Carrasco Pradas, A., Gracia Expósito, E. y De la Iglesia Villasol, C. (2005). Las TIC en la construcción del Espacio Europeo de Educación Superior: Dos experiencias docentes en teoría económica. Revista Iberoamericana de Educación, vol. 36, n. 1, 1-16.

Cassany, D. (1996). La cocina de la escritura. Barcelona: Anagrama. Recuperado de http:// ifdc6m.juj.infd.edu.ar/aula/archivos/repositorio/250/269/Cassany_D_-_La_Cocina_De_La_ Escritura.doc, consultado en marzo de 2013.

Cochran-Smith, M. (1991). Word Processing and Writing in Elementary Classrooms: A Critical Review of Related Literature. Review of Educational Research, vol. 61, n. 1, 107-155.

Collier, R. \& Werier, C. (1995). When Computer Writers Compose by Hand. Computers and Composition, vol. 12, n. 1, 47-59.

Corcoran, T. \& Silander, M. (2009). Instruction in High Schools: The Evidence and the Challenge. Future of Children, vol. 19, n. 1, 157-183.

Cox, M. J., Cox, K. \& Preston, C. (2000). What Factors Support or Prevent Teachers from Using ICT in their Classrooms? British Educational Research Association Annual Conference, University of Sussex at Brighton, United Kingdom. Recuperado de http://www.leeds.ac.uk/educol/ documents/00001304.html, consultado en marzo de 2013.

De Barrera, L. F. (2003). Pedagogía integradora en el aula: Teoría, práctica y evaluación de estrategias de adquisición de competencias cognitivas y lingüísticas para el empleo efectivo de la lengua materna oral y escrita. El Nacional: Caracas.

De Smet, M. J. R., Brand-Gruwel, S., Broekkamp, H. \& Kirschner, P. A. (2012). Write between the Lines: Electronic Outlining and the Organization of Text Ideas. Computers in Human Behavior, vol. 28, n. 6, 2107-2116.

Dybdahl, C. S., Shaw, D. G. \& Blahous, E. (1997). The Impact of the Computer on Writing. Computers in the Schools, vol. 13, n. 3, 41-53.

Ferreiro, E. y Teberosky, A. (1999). Los sistemas de escritura en el desarrollo del niño. México D.F.: Siglo XXI.

(1981). La comprensión del sistema de escritura: Construcciones originales del niño e información específica de los adultos. Lectura y Vida, n. 1. Recuperado de http://www.lecturayvida. fahce.unlp.edu.ar/numeros/a2n1/02_01_Ferreiro.pdf, consultado en marzo de 2013.

Ferris, S. P. (2002). Writing Electronically: The Effects of Computers on Traditional Writing. The Journal of Electronic Publishing, vol. 8, n. 1. Recuperado de http://quod.lib.umich.edu/cgi/t/text/ text-idx?c=jep;view=text;rgn=main;idno=3336451.0008.104, consultado en febrero de 2013.

Flower, L. \& Hayes, J. (1996). Textos en contexto. Los procesos de lectura y escritura. Buenos Aires: Asociación Internacional de Lectura. Lectura y Vida. Recuperado de https://ciie-r10.wikispaces. $\mathrm{com} /$ file/view/Flowers $+\mathrm{y}+$ Hayes $+\mathrm{La}+$ teoria+de+la+redaccion+como+proceso+cognitivo.pdf, consultado en febrero de 2013.

(1981). A Cognitive Process Theory of Writing. College Composition and Communication, vol. 32, n. 4, 365-387.

Goldberg, A., Russell, M. \& Cook, A. (2003). The Effect of Computers on Student Writing: A Meta-analysis of Studies from 1992 to 2002. Journal of Technology, Learning, and Assessment, vol. 2, n. 1, 1-52.

Grigar, D. (2005). The Challenges of Hybrid Forms of Electronic Writing. Computers and Composition, vol. 22, n. 3, 375-393.

Guzmán, R. (2004). Producción infantil de textos expositivos. Revista Educación y Educadores, n. 7, 157-175.

Jolibert, J. y Jacob, J. (1998). Interrogar y producir textos auténticos: Vivencias en el aula. 
Santiago: Dolmen Ediciones.

Karsenti, T. y Lira-Gonzales, M. L. (2011). La importancia de la motivación y las habilidades computacionales de los futuros profesores en el uso de las TIC. Revista Iberoamericana de Educación Superior, vol. 3, n. 8. Recuperado de http://ries.universia.net/index.php/ries/article/viewArticle/87, consultado en marzo de 2013.

Lunsford, K. (2006). Computer-Supported Writing. In K. Brown (Ed.), Encyclopedia of Language \& Linguistics $\left(2^{\text {nd }}\right.$ Ed.) (pp. 809-816). Oxford: Elsevier.

Lyman, P. (1984). Reading, Writing and Word Processing: Toward a Phenomenology of the Computer Age. Qualitative Sociology, vol. 7, n. 1, 75-89.

Mogey, N. \& Hartley, J. (2013). To Write or to Type? The Effects of Handwriting and Wordprocessing on the Written Style of Examination Essays. Innovations in Education and Teaching International, vol. 50, n. 1, 85-93.

, Cowan, J., Paterson, J. \& Purcell, M. (2012). Students' Choices between Typing and Handwriting in Examinations. Active Learning in Higher Education, vol. 13, n. 2, 117-128. , Paterson, J., Burk, J. \& Purcell, M. (2010). Typing Compared with Handwriting for Essay Examinations at University: Letting the Students Choose. ALT-J: Research in Learning Technology, vol. 18, n. 1, 29-47.

., Sarab, G., Haywood, J., Van Heyningen, S., Dewhurst, D., Hounsell, D. \& Neilson, R. (2008). The End of Handwriting? Using Computers in Traditional Essay Examinations. Journal of Computer Assisted Learning, vol. 24, n. 1, 39-46.

Moran, C. (1983). Electronic Media: Word Processing and the Teaching of Writing. The English Journal, vol. 72, n. 3, 113-115.

Morphy, P. \& Graham, S. (2012). Word Processing Programs and Weaker Writers/Readers: A Meta-analysis of Research Findings. Reading and Writing, vol. 25, n. 3, 641-678.

Olson, C. B., Land, R., Anselmi, T. \& Aubuchon, C. (2010). Teaching Secondary English Learners to Understand, Analyze, and Write Interpretive Essays about Theme. Journal of Adolescent and Adult Literacy, vol. 54, n. 4, 245-256.

Passey, D., Rogers, C., Machell, J., McHugh, G. \& Allaway, D. (2004). The Motivational Effect of ICT on Pupils. Research Project 4RP/2002/050-3. Recuperado de http://www.canterbury.ac.uk/ education/protected/spss/docs/motivational-effect-ict-brief.pdf, consultado en marzo de 2013.

Pennington, M. C. (1991). Positive and Negative Potentials of Word Processing for ESL Writers. System, vol. 19, n. 3, 267-275.

Portolés, J. (1993). La distinción entre los conectores y otros marcadores del discurso en español. Verba, n. 20, 141-170.

Prieto Cortés, M. L. (2012). Una mirada pedagógica a problemas en el aprendizaje de la lectura y escritura diagnosticados en niños de 5 a 6 años de edad por disciplinas de apoyo como la terapia ocupacional. Tesis de Maestría en Pedagogía, Universidad de La Sabana, Colombia. Recuperado de http://intellectum.unisabana.edu.co:8080/jspui/handle/10818/3628, consultado en octubre de 2012.

Regan, K., \& Berkeley, S. (2012). Effective Reading and Writing Instruction: A Focus on Modeling. Intervention in School and Clinic, vol. 47, n. 5, 276-282.

Russell, M., \& Haney, W. (1997). Testing Writing on Computers: An Experiment Comparing Student Performance on Tests Conducted via Computer and via Paper-and-pencil. Education Policy Analysis Archives, vol. 5, n. 3. Recuperado de http://epaa.asu.edu/ojs/article/view/604/726, consultado en febrero de 2013.

Sag-Legrán, P. (2010). La Competencia Lingüística. Innovación y Experiencias Educativas, n. 27. Recuperado de http://www.csi-csif.es/andalucia/modules/mod_ense/revista/pdf/Numero_27/ PETRA_SAG_LEGRAN_01.pdf, consultado en febrero de 2013.

Salinas Herrera, J. (2004). Innovación docente y uso de las TIC en la enseñanza universitaria. Revista Universidad y Sociedad del Conocimiento, vol. 1, n. 1. Recuperado de http://www.uoc.edu/ rusc/dt/esp/salinas1104.pdf, consultado en marzo de 2013. 
Sánchez, A. (2006). Prevención de las dificultades en lectura y escritura “Juanita Enseña”. Chía: Universidad de La Sabana.

Scardamalia, M., Bereiter, C. \& Steinbach, R. (1984). Teachability of Reflective Processes in Written Composition. Cognitive Science, vol. 8, n. 2, 173-190.

Takayoshi, P. (1996). The Shape of Electronic Writing: Evaluating and Assessing Computerassisted Writing Processes and Products. Computers and Composition, vol. 13, n. 2, 245-257.

Tejada Fernández, J., Navío Gámez, A. y Ruiz Bueno, C. (2007). La didáctica de un entorno virtual interuniversitario: Experimentación de ECTS apoyados en TIC. Pixel-Bit: Revista de Medios y Educación, n. 30, 95-118.

Villalón, M., Rojas-Barahona, C. A., Förster, C. E., Valencia, E., Cox, P. y Volante, P. (2011). Resultados de la enseñanza de estrategias de lectura y escritura en la alfabetización temprana de niños con riesgo social. Estudios Sobre Educación, n. 21, 159-179.

Whithaus, C., Harrison, S. B. \& Midyette, J. (2008). Keyboarding Compared with Handwriting on a High-stakes Writing Assessment: Student Choice of Composing Medium, Raters' Perceptions, and Text Quality. Assessing Writing, vol. 13, n. 1, 4-25.

Zinna, A. (2011). The Object of Writing. Language Sciences, vol. 33, n. 4, 634-646.

Zvacek, S. M. (1988). Word Processing and the Teaching of Writing. Computers in Human Behavior, vol. 4, n. 1, 29-35. 
